\title{
Effect of Implementing Nursing Guideline on Critically Patients Outcomes' Regarding Sedation
}

\author{
Heba Hussein Gaber ${ }^{1}$, Alaa Mohamed Ahmed ${ }^{2}$, Mogedda Mohamed Mehany ${ }^{3}$ \& Asmaa Aly Mahgoub ${ }^{4}$. \\ 1. Nurse Specialist in Assuit University Hospital, Egypt. \\ 2. Professor of Anthesia and ICU Faculty of Medicine, Assuit University Egypt \\ 3. Assistant Professor of Critical Care \& Emergency Nursing Faculty of Nursing, Assuit University Egypt. \\ 4. Lecture of Critical Care \& Emergency Nursing Faculty of Nursing, Assuit University Egypt.
}

\begin{abstract}
Sedation can help alleviate pain of intrusion allows patients to tolerate painful/distressing procedures optimize mechanical ventilation used to decrease $\mathrm{O} 2$ consumption, decrease ICP in neurosurgical patient,and facilitate cooling Control agitation. Reduce anxiety to alleviate pain, for amnesia during neuromuscular blocked. Aim of the present study was to evaluate the effect of implementing nursing guideline on critically patients' outcomes regarding sedation in ICU at Assiut University Hospitals. Design: Quesi experimental design was used to conduct the aim of this study. Subjects: A purposive sample of 60 adult's critically ill patients from both Sexes admitted in the general intensive care unit at Assiut University hospital Tools: Tool I: Patient assessment sheet, Tool II: Sedative assessment scales Method: the researcher used the study toolsand implement nursing guideline for study group. Results: finding of the present study revealed that there was a signifficant statistical diffrences between both study and control groups in relation to sedative complication tracheostomy, long ICU stay, and mortality ( $\mathrm{p}<0.05$ ) Conclusion: implementation of nursing guidline about sedation was significantly effective in improve outcomes of critically sedative patients. Recomendation: emphasize to utilize the nursing guidline about sedation in the critically care units
\end{abstract}

\section{Key wards: Critically Ill Patient, Nursing Guidelines, Patient Out Comes \& Sedation.}

\section{Introduction}

Sedation is important in the ICU to facilitate amnesia during critical illness, to prevent delirious patients from causing harm to self and others, to facilitate invasive management, to promote ventilator-patient synchrony, to circumvent post-traumatic stress disorder and to relieve dyspnea. Inability to meet goals of proper sedation and analgesia has deleterious consequences in the form of increase in adverse events, poor overall outcomes, longer ICU stays and economic effects. Analgesia is as important a component of ICU patients as is sedation. An "analgesia-first" approach can prove beneficial in agitated or delirious patients. (Hariharan, et al., 2017)

Sedation in critically ill patient has changed dramatically over time for many years; the patient in the ICU was deeply sedated and immobile. After years of research, it was concluded that this deep sedation was not beneficial for the patient in fact, it produced a number of complications the deeply sedated patient was unable to move or shift positions, leading to an increased risk of pressure sore development. Patients who were mechanically ventilated in the control mode were difficult to wean from the ventilator when the appropriate time came, prolonged ICU stays, acute brain dysfunction, and an increased risk of death. In addition, family member were unable to interact with their loved ones, leading to a significant emotional impact upon these individuals. (Norman, et al.., 2014).

The American Nurses Association recommended that critical care nurses who administer and monitor procedural sedation and analgesia are able to identify and differentiate the various levels of sedation; demonstrate the acquired knowledge of anatomy, physiology, pharmacology, cardiac dysrhythmia recognition; detect complications related to moderate analgesia and sedation and appropriately intervene; demonstrate competence in pre-procedural, procedural, and post-procedural nursing care from the initial patient evaluation to patient discharge; anticipate, recognize, and address potential complications during the process; and understand the medico-legal aspects of procedural analgesia and sedation. (Varndell, et al., 2015).

The goal of these clinical practice guidelines is to recommend best practices for managing pain, agitation and delirium (PAD) to improve clinical outcomes in adult ICU patients.

\section{Significance of study}

The need for sedative therapy in critical care adults receiving mechanical ventilation is well established; $85 \%$ of intensive care unit (ICU) patients are given intravenous sedatives to help attenuate the anxiety, pain, and agitation associated with mechanical ventilation. the overall goals of the sedation are to 
provide stability in physiological status and comfort. (Grape \& Cindyl 2012).

Statistical report of general ICU' pharmacy at Assiut University Hospital in (2014-2015) documented that 5200 ampoule from midazolam (each ampule contain $3 \mathrm{ml}$ each $1 \mathrm{ml}$ contain $5 \mathrm{mg}$ ) and 2600 ampoule from propofol (each ampule contain $20 \mathrm{ml}$ each $1 \mathrm{ml}$ contain $10 \mathrm{mg}$ ) was given to patients.

\section{Aim of study}

The current study aimed to evaluate the effect of implementing nursing guideline on critically patients' outcomes regarding sedation (tracheostomy, resedationand over sedation, mortality, and ICUstaying) in ICU at Assiut University Hospitals.

\section{Subjects \& Method \\ Research design:}

A quesi -experimental design was used to conduct the aim of this study.

\section{Setting}

The study was carried out in general intensive care unit (18 beds) in general Assuit University Hospital.

\section{Sampling}

A purposive sample of 60 patients from both sex adult critically patients admitted in the previously mentioned setting who received sedative agent available at the time of data collection these patients divided in to two group (30 in each) control \& study groups.

\section{Research hypothesis:}

To fulfill the aim of the study the following research hypothesis was formulated

1- The post mean of the study group subjects will better than that of control groups one:

2- The post mean length of hospital of patient will be exposed to the designed nursing guideline protocol will be lesser than their pre mean length of hospital stay of the control group.

3- The frequency complication for patients who will be exposed to the designed nursing guide line for sedative critically patient protocol will be lesser than among control group patients.

\section{Inclusion criteria}

- Both sexes.

- Adult (18-60 yrs).

- Patients received sedation for 3 day.

Exclusion criteria

The study excluded patients who had the following criteria:-

- Comatosed patient

- Patient with neuromuscular diseases.

- Planned weaning from mechanical ventilation.
Tools

Two tools were used by the researcher in the present study after reviewing of the related literature (Morgan 2007, Hogarth \& Hall 2004, Grap 2012, Sherman \& de beer 2014, \& Richard 2013).

First tool: Patient assessment tool

This tool developed by the researcher after reviewing related literature this tool to assess social \& clinical data which includes three parts.

\section{Personal \&clinical data}

Part I: socio - demographic data which includes patient code ,sex , age, and date of admission, date of discharge and health relevant data that include patient diagnosis on admission, chronic diseases history, surgical performance more over assess the type of sedation ,name of sedation, dose, aim of sedation and other medication which patient received .

Part II: assessment of hemodynamic status this part was used to assess hemodynamic status which includes central venous pressure, heart rate, respiratory rate taken from bed side monitor.

The $2^{\text {nd }}$ tool Sedative assessment scales tool These tools adopted from review (Morgan 2007, Sherman, De beer 2014, \& stites 2013) and include 4 parts:

Part I: Behavioral pain scales which includes 3 items (facial expression, upper extremities and compliance with ventilation) Score ranges from 3 (no pain) to 12 (maximum pain).

Part II: Richmond agitation sedation scale (RASS) was adopted from (Sherman, De beer2014) and it was used to assess the effect of sedation and measurement of consciousness in 10 scores. (From 4 to -5 point) the RASS has the unique feature of measuring length of eye contact by the patient following verbal command. - Patient is alert, restless, or agitated. (Score 0 to +4 )

- Patient awakens with sustained eye opening and eye contact (Score -1$)$.

- Patient awakens with eye opening and eye contact, but not sustained.

(Score-2)

- Patient has any movement in response to voice but no eye contact. (Score -3) - Patient has any movement to physical stimulation. (Score -4$)$

- Patient has no response to any stimulation. (Score 5)

Part III: ICU delirium screening checks list (ICDSC) is the most valid and reliable delirium monitoring tool in adult ICU patients

The ICDSC was an 8 item check list that it designed to be completed based on data from the previous 24 hours the 8 items are scored 1 (present) or 0 (absent) A score of 4 or greater is positive score of 1 $3=$ subsysndromal delirium score of $>$ or $=4$ delirium. 


\section{Method:}

The study was conducted through main phases, which are preparatory, implementation and evaluation phases:-

\section{Preparatory phase}

1. Permission to conduct the study was obtained from the hospital responsible authorities in anesthesiology department, general intensive care unit after explanation of the aim and natural of the study.

2. The tools (II) used in this study were adopted by the researcher based on reviewing the relevant literature.

3. The tool was tested for content related validity by 5 specialists in the field three of critical care nursing and two critical care medicines.

4. A pilot study was conducted on 5 sedative patients to assess the feasibility and applicability of the tool and the necessary modification were done.

5. The Reliability was done on tool one using Cronach's Alpha and reliability level was 0.87 to assess the consistency and stability of the tools which was accepetable.

\section{Ethical considerations}

1. Research proposal was approved from Ethical committee in the Faculty of nursing.

2. There is no risk for study subject during application of the research.

3. The study was following common ethical principles in clinical research.

4. Written consent was obtained from patients or guidance that is willing to participate in the study, after explaining the nature and purpose of the study.

5. Confidentiality and anonymity was assured.

6. Study subject have the right to refuse to participate and or withdraw from the study without any rationalat any time.

7. Study subjects privacy was considered during collection of data.

Data collection

- Data collection in one year from Septamber 2016 to Septamber 2017.

- The data collected from the first day when the patients receive the sedation drug.

- For three days every day and every shift the data were recorded in the developed tools.

- The researcher assigned the study sample (60 patients) to two groups (control group and study group).

- For the control group: the researcher assessed patients who were receiving the routine hospital care for sedated patients.
- For study group: the researcher assesses the patient then applying care of nursing guidelines for sedated patients.

\section{Assessment phase for both groups}

- During this phase the patient assessed Form the first day of starting sedation and record patient socio demographic and clinical data by taking this information from his or her sheet using tool 1( part I).

- The researcher assessed homodynamic state of patient before starting sedation and during and after withdrawal from sedation using tool1 (part II).

- The researcher assesses the patients using tool 2 (part I) to determine the degree of pain by behavioral pain assessment scale one time before starting, during and after sedation.

- The researcher assesses the patients using tool 2(part II) Richmond agitation sedation scale to determine the quality and depth of sedation and the effect of the drug in treating the agitation one time before, during and after sedation induction.

- The researcher assesses the patients using tool2 (part III) to monitor delirium by intensive screening checklist one time before, during and after sedation.

Implementation phase for study group

The researcher applied the guide lines about sedation as the follow:

- Apply the daily awaking trail to minimizethe effect of sedation in criticaly ill patients, while maintaining adequate pain control.

o Discuss with Intensivist daily awaking trail.

o Explain thedaily awaking trail to patient and his /her family.

o Put the patient in comfort postion.

o Stocked crash cart.

Daily awaking trail technique

1- Take permission from the doctor to start daily awaking trail.

2- The critical care nursing before starting daily awaking trail perform safty screen prior to sedation lightening process.

3- Stop all sedative agents and don't stop analgesia to control pain if present.

4- Assess neurological status and perform intensive delirium screening check list.

5- If daily awaking trail sucses or Pass and manifested by Patients open his/her eyes to verbal stimuli and follow simple commands (i.e. squeeze hand, track with eyes, and stick out tongue).

- Continually reorient the patient with targeting the lightest possible level of sedation.

- Promote sleep in ICU by controlling light and minimize noise Perform early mobilization every day after patients were awake and able to follow the instructions. 
- Perform timely removal of catheters and physical restraint and ensure the use of eye glasses magnifying lenses and hearing aid and encourage communication between patient and his /her near of family.

If daily awaking trail fail—STOP daily awaking trail process and discuss findings with Intensivist team.

\section{Daily awaking trail failure criteria}

- O2 saturation $\leq 88 \%$ for greater than 5 minutes.

- Sustained anxiety, agitationsor Richmond agitation scores: $\mathrm{RASS} \geq+2$.

- Respiratory distress for greater than 5 minutes; rate $\geq 35 / \mathrm{min}$.

- Acute cardiac arrhythmia.

- New onset diaphoresis.

- Increased ICP.

Evaluation phase

This phase was done to evaluate the effect of applying nursing guidelines about sedation on study groupthree time before sedation, during and after withdrawal of sedation by using tool two to evaluate patients out comes, hospital stay and complication of sedation.

\section{Statistical analysis}

- The data were reviewed and prepared for computer entry, coded, analyzed and tabulated and using computer program (SPSS Iversion 22)

- Descriptive statistics' such as frequencies and percentage, mean and standard deviation.

- Independent sample $\mathrm{T}$ test and one way ANOVA test used in the relation ship between study and control groups.

- The critical value of the tests $\mathrm{P}$ was considered statistically significant when $\mathrm{P}$ less than 0.05 .

- Cronbachs alpha was done to test reliability of the tool.

\section{Results}

Table (1): Distribution of sample related to socio-demographic data.

\begin{tabular}{|c|c|c|c|c|c|}
\hline \multirow{2}{*}{ Items } & \multicolumn{2}{|c|}{ Control Group } & \multicolumn{2}{|c|}{ Study Group } & \multirow{2}{*}{ P-value } \\
\hline & No. $(n=30)$ & $\%$ & No. $(n=30)$ & $\%$ & \\
\hline Age: & & & & & \multirow{2}{*}{0.896} \\
\hline Mean \pm SD & \multicolumn{2}{|c|}{$39.6 \pm 12.2$} & \multicolumn{2}{|c|}{$39.2 \pm 11.3$} & \\
\hline \multicolumn{5}{|l|}{ Gender: } & \multirow{3}{*}{0.182} \\
\hline Male & 19 & 63.3 & 18 & 83.3 & \\
\hline Female & 11 & 36.7 & 12 & 16.7 & \\
\hline \multicolumn{5}{|l|}{ Chronic diseases: } & \multirow{7}{*}{0.191} \\
\hline Yes & 13 & 43.3 & 8 & 26.7 & \\
\hline Heart disease & 3 & 10.0 & 1 & 3.3 & \\
\hline D.M & 3 & 10.0 & 4 & 13.4 & \\
\hline Hypertension & 4 & 10.0 & 0 & 0.0 & \\
\hline Liver diseases & 3 & 6.7 & 0 & 0.0 & \\
\hline No & 17 & 56.6 & 22 & 73.3 & \\
\hline \multicolumn{5}{|l|}{ Degree of sedation: } & \multirow{4}{*}{0.181} \\
\hline Mild & 7 & 23.3 & 6 & 20.0 & \\
\hline Moderate & 17 & 56.7 & 20 & 66.7 & \\
\hline Deep & 6 & 20.0 & 4 & 13.34 & \\
\hline \multicolumn{5}{|l|}{ Aim of sedation: } & \multirow{4}{*}{0.222} \\
\hline Agitation & 6 & 20.0 & 9 & 30.0 & \\
\hline Pain & 8 & 26.7 & 12 & 40.0 & \\
\hline Mechanical ventilation & 16 & 53.3 & 9 & 30.0 & \\
\hline
\end{tabular}

Independent sample T-test

* Statistical significant differences $(p<0.05)$ 


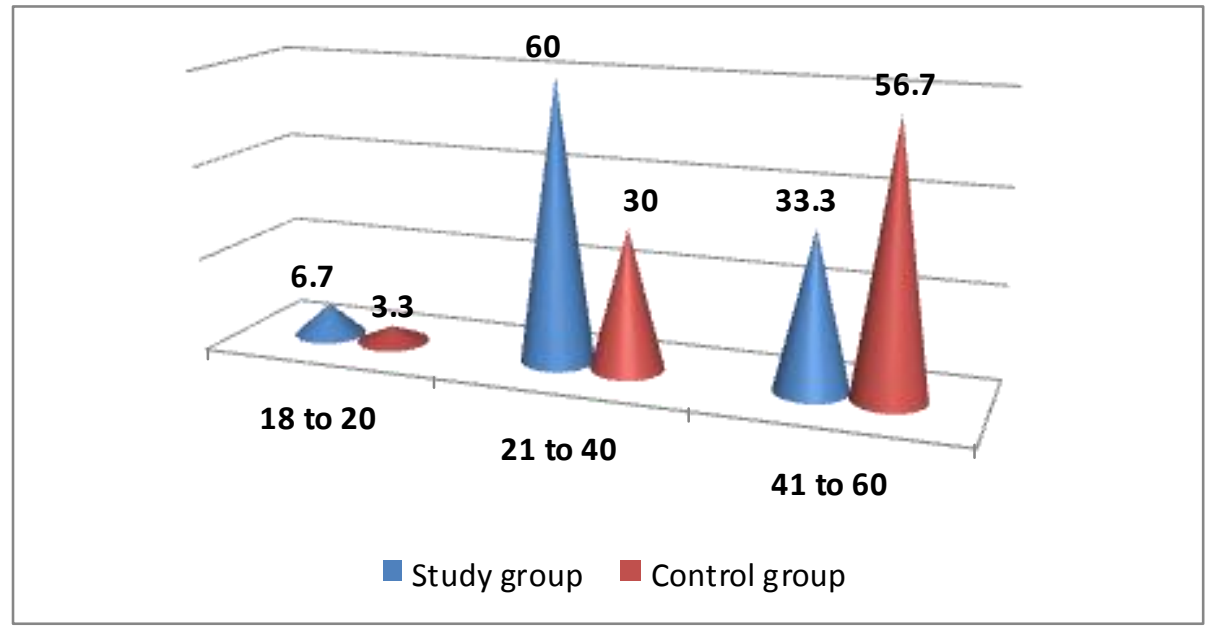

Figure (1): Percentages distribution of studied patients according to age.

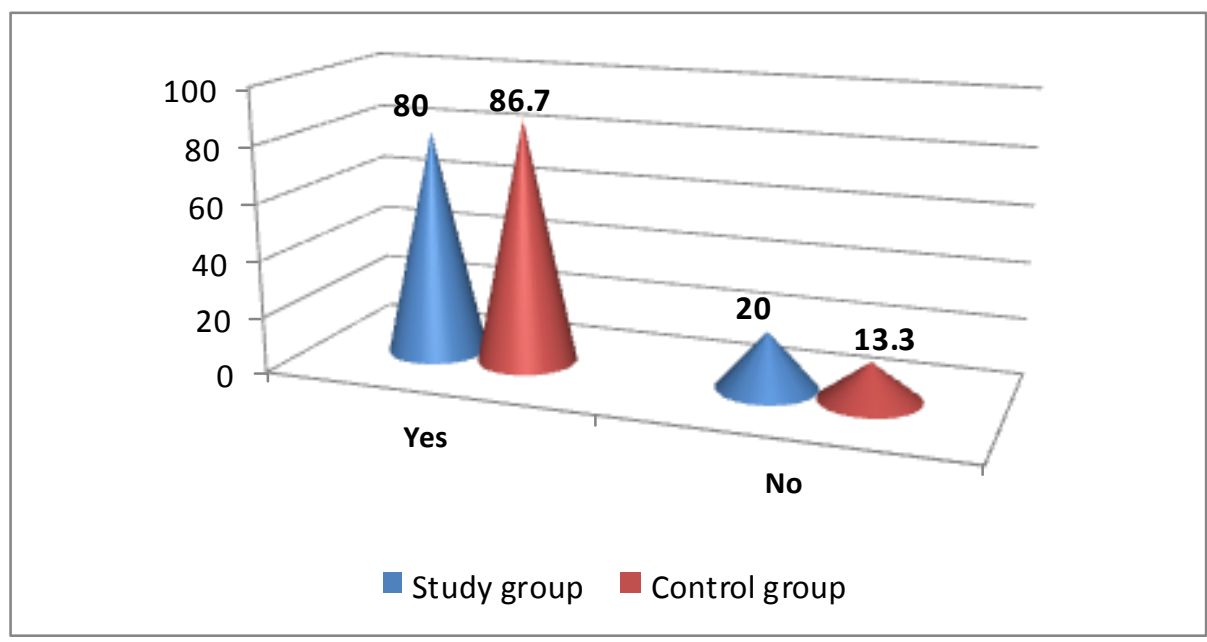

Figure (2): Percentages distribution of studied patients according to surgical performance.

Table (2): Percentage distribution of patients' behavioral pain scale.

\begin{tabular}{|c|c|c|c|c|c|}
\hline \multirow{2}{*}{ Patients' behavioral pain scale } & \multicolumn{2}{|c|}{$\begin{array}{c}\text { Control group } \\
(\mathbf{n}=\mathbf{3 0})\end{array}$} & \multicolumn{2}{|c|}{$\begin{array}{c}\text { Study group } \\
(\mathbf{n}=\mathbf{3 0})\end{array}$} & \multirow{2}{*}{ P-value } \\
\hline & No. & $\%$ & No. & $\%$ & \\
\hline \multicolumn{5}{|l|}{ Before starting sedation } & \multirow{6}{*}{$0.001 * *$} \\
\hline No Pain $(<=3)$ & 0 & 0.0 & 0 & 0.0 & \\
\hline Slight pain (4 to 6) & 5 & 16.7 & 0 & 0.0 & \\
\hline Moderate pain (7 to 9 ) & 1 & 3.3 & 16 & 53.3 & \\
\hline Extreme pain (10 to 12) & 24 & 80.0 & 14 & 46.7 & \\
\hline Mean \pm SD & \multicolumn{2}{|c|}{$9.3 \pm 1.5$} & \multicolumn{2}{|c|}{$9.8 \pm 1.1$} & \\
\hline \multicolumn{5}{|l|}{ During sedation } & \multirow{6}{*}{$0.049 *$} \\
\hline No Pain $(<=3)$ & 0 & 0.0 & 0 & 0.0 & \\
\hline Slight pain (4 to 6) & 8 & 26.7 & 16 & 53.3 & \\
\hline Moderate pain (7 to 9) & 22 & 4.3 & 14 & 46.7 & \\
\hline Extreme pain (10 to 12$)$ & 0 & 0.0 & 0 & 0.0 & \\
\hline Mean \pm SD & \multicolumn{2}{|c|}{$7.4 \pm 1.8$} & \multicolumn{2}{|c|}{$7.1 \pm 1.3$} & \\
\hline After withdrawal & & & & & $0.028^{*}$ \\
\hline
\end{tabular}




\begin{tabular}{|c|c|c|c|c|c|}
\hline \multirow[t]{2}{*}{ Patients' behavioral pain scale } & \multicolumn{2}{|c|}{$\begin{array}{c}\text { Control group } \\
(\mathbf{n}=\mathbf{3 0})\end{array}$} & \multicolumn{2}{|c|}{$\begin{array}{c}\text { Study group } \\
(\mathbf{n}=\mathbf{3 0})\end{array}$} & \multirow[t]{6}{*}{ P-value } \\
\hline & No. & $\%$ & No. & $\%$ & \\
\hline No Pain $(<=3)$ & 26 & 86.7 & 30 & 0.0 & \\
\hline Slight pain (4 to 6) & 4 & 13.3 & 0 & 0.0 & \\
\hline Moderate pain (7 to 9) & 0 & 0.0 & 0 & 0.0 & \\
\hline Extreme pain (10 to 12$)$ & 0 & 0.0 & 0 & 0.0 & \\
\hline Mean \pm SD & \multicolumn{2}{|c|}{$3.1 \pm 0.9$} & \multicolumn{2}{|c|}{$3.0 \pm 0.0$} & \\
\hline
\end{tabular}

One way ANOVA

* Statistical significant differences $(p<0.05)$

Table (3): Percentage distribution of patients' Richmond agitation sedation scale.

\begin{tabular}{|c|c|c|c|c|c|}
\hline & \multicolumn{2}{|c|}{$\begin{array}{c}\text { Control group } \\
(\mathbf{n = 3 0})\end{array}$} & \multicolumn{2}{|c|}{$\begin{array}{c}\text { Study group } \\
(\mathrm{n}=\mathbf{3 0})\end{array}$} & \multirow[t]{2}{*}{ P-value \% } \\
\hline & No. & $\%$ & No. & No. & \\
\hline \multicolumn{5}{|c|}{ Before starting sedation } & \multirow{12}{*}{0.359} \\
\hline Combative+4 & 0 & 0.0 & 0 & 0.0 & \\
\hline Very agitated +3 & 16 & 53.3 & 18 & 60.0 & \\
\hline Agitated +2 & 12 & 40.0 & 12 & 40.0 & \\
\hline Restless+1 & 2 & 6.7 & 0 & 0.0 & \\
\hline Alert and calm0 & 0 & 0.0 & 0 & 0.0 & \\
\hline Drowsy-1 & 0 & 0.0 & 0 & 0.0 & \\
\hline Light sedation-2 & 0 & 0.0 & 0 & 0.0 & \\
\hline Moderate sedation -3 & 0 & 0.0 & 0 & 0.0 & \\
\hline Deep sedation -4 & 0 & 0.0 & 0 & 0.0 & \\
\hline Un araousable-5 & 0 & 0.0 & 0 & 0.0 & \\
\hline Mean \pm SD & \multicolumn{2}{|c|}{$2.4 \pm 0.6$} & \multicolumn{2}{|c|}{$2.6 \pm 0.4$} & \\
\hline \multicolumn{5}{|l|}{ During sedation } & \multirow{12}{*}{$0.001 * *$} \\
\hline Combative+4 & 0 & 0.0 & 0 & 0.0 & \\
\hline Very agitated +3 & 0 & 0.0 & 0 & 0.0 & \\
\hline Agitated +2 & 4 & 13.3 & 8 & 26.7 & \\
\hline Restless+1 & 4 & 13.3 & 8 & 26.7 & \\
\hline Alert and calm0 & 0 & 0.0 & 0 & 0.0 & \\
\hline Drowsy-1 & 2 & 6.7 & 12 & 40.0 & \\
\hline Light sedation-2 & 7 & 23.3 & 2 & 6.6 & \\
\hline Moderate sedation -3 & 11 & 36.7 & 0 & 0.0 & \\
\hline Deep sedation -4 & 2 & 6.4 & 0 & 0.0 & \\
\hline Un araousable-5 & 0 & 0.0 & 0 & 0.0 & \\
\hline Mean \pm SD & \multicolumn{2}{|c|}{$-1.5 \pm 1.9$} & \multicolumn{2}{|c|}{$0.2 \pm 1.4$} & \\
\hline \multicolumn{5}{|l|}{ After withdrawal } & \multirow{12}{*}{$0.005 * *$} \\
\hline Combative+4 & 0 & 0.0 & 0 & 0.0 & \\
\hline Very agitated +3 & 0 & 0.0 & 0 & 0.0 & \\
\hline Agitated +2 & 0 & 0.0 & 0 & 0.0 & \\
\hline Restless+1 & 2 & 6.7 & 4 & 13.3 & \\
\hline Alert and calm0 & 18 & 60.0 & 18 & 60.0 & \\
\hline Drowsy-1 & 2 & 6.7 & 8 & 26.7 & \\
\hline Light sedation-2 & 0 & 0.0 & 0 & 0.0 & \\
\hline Moderate sedation -3 & 0 & 0.0 & 0 & 0.0 & \\
\hline Deep sedation -4 & 0 & 0.0 & 0 & 0.0 & \\
\hline Un araousable-5 & 8 & 26.7 & 0 & 0.0 & \\
\hline Mean \pm SD & \multicolumn{2}{|c|}{$-1.3 \pm 2.2$} & \multicolumn{2}{|c|}{$-1.3 \pm 0.6$} & \\
\hline
\end{tabular}

One way ANOVA

* Statistical significant differences $(p<0.05)$ 
Table (4): Percentage distribution of patients' ICU screening check list for delirium.

\begin{tabular}{|c|c|c|c|c|c|}
\hline \multirow{2}{*}{ Patients' ICU screening check list for delirium scale } & \multicolumn{2}{|c|}{$\begin{array}{c}\text { Control group } \\
(\mathbf{n}=\mathbf{3 0})\end{array}$} & \multicolumn{2}{|c|}{$\begin{array}{c}\text { Study group } \\
(\mathbf{n}=\mathbf{3 0})\end{array}$} & \multirow{2}{*}{ P-value } \\
\hline & No. & $\%$ & No. & $\%$ & \\
\hline \multicolumn{5}{|l|}{ Before starting sedation } & \multirow{5}{*}{0.087} \\
\hline Normal (0) & 15 & 50.0 & 23 & 76.7 & \\
\hline Subsyndromal (1 to 3 ) & 15 & 50.0 & 7 & 23.3 & \\
\hline Delirium $(>=4)$ & 0 & 0.0 & 0 & 0.0 & \\
\hline Mean \pm SD & \multicolumn{2}{|c|}{$0.9 \pm 1.1$} & \multicolumn{2}{|c|}{$0.3 \pm 0.7$} & \\
\hline During sedation & & & & & \multirow{5}{*}{$\begin{array}{c}0.001 \\
* *\end{array}$} \\
\hline Normal (0) & 19 & 63.3 & 4 & 13.3 & \\
\hline Subsyndromal (1 to 3 ) & 7 & 23.3 & 24 & 80.0 & \\
\hline Delirium $(>=4)$ & 4 & 13.4 & 2 & 6.7 & \\
\hline Mean \pm SD & \multicolumn{2}{|c|}{$1.1 \pm 1.9$} & \multicolumn{2}{|c|}{$2.4 \pm 1.1$} & \\
\hline After withdrawal & & & & & \multirow{5}{*}{$\begin{array}{l}0.006 \\
* *\end{array}$} \\
\hline Normal (0) & 19 & 63.3 & 30 & 100 & \\
\hline Subsyndromal (1 to 3$)$ & 9 & 30.0 & 0 & 0.0 & \\
\hline Delirium $(>=4)$ & 2 & 6.7 & 0 & 0.0 & \\
\hline Mean \pm SD & \multicolumn{2}{|c|}{$1.2 \pm 2.1$} & \multicolumn{2}{|c|}{$0.0 \pm 0.0$} & \\
\hline
\end{tabular}

One way ANOVA $\quad *$ Statistical significant differences $(p<0.05)$

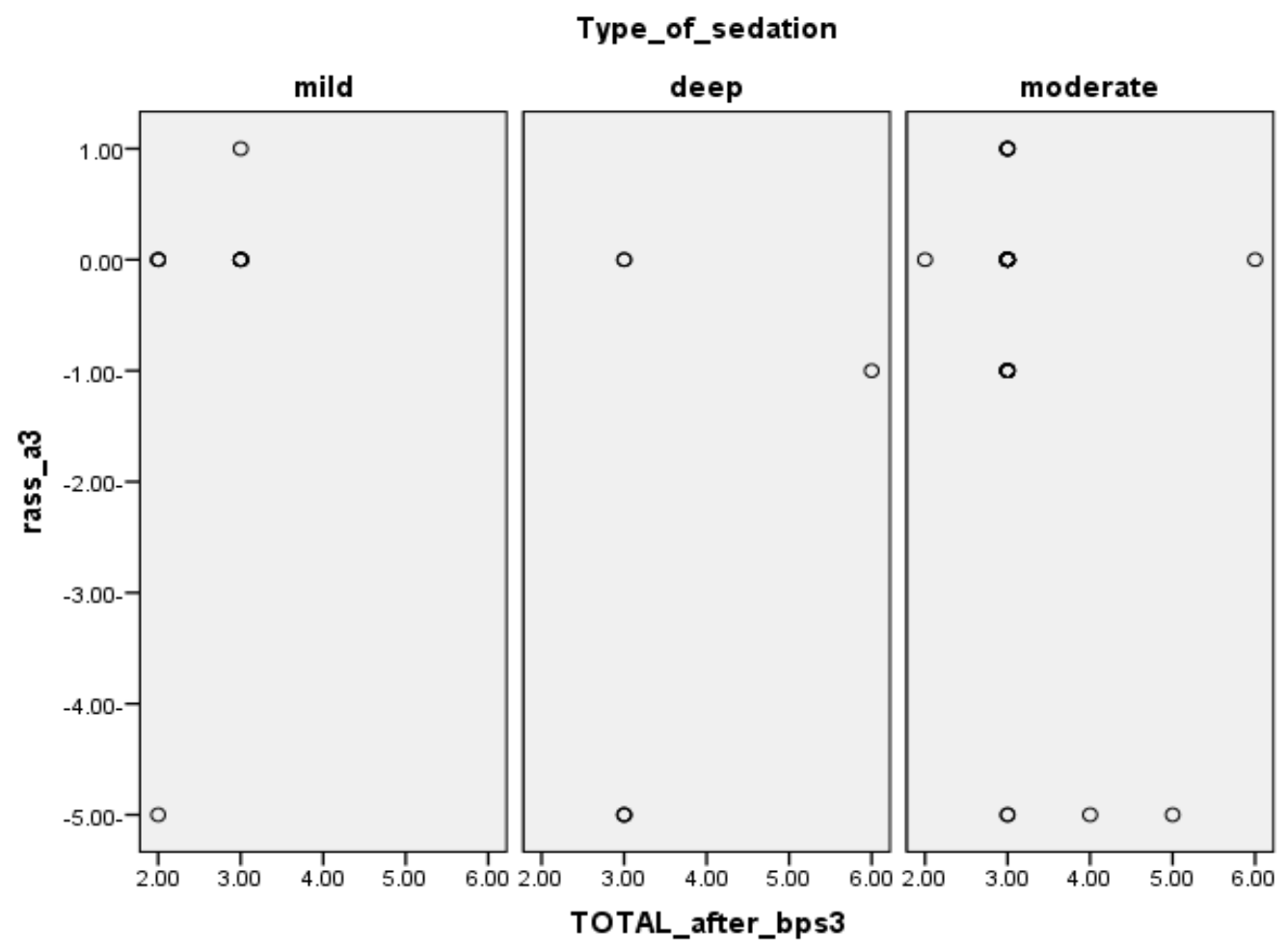

Figure (3): Correlation between patients' behavioral pain scale and Richmond agitation sedation scale related

This figure shows that:

to type of sedation

Statistical significance difference between behavioral pain scale and Richmond agitation sedation scale related to type of sedation 
Table (5): Percentage distribution of patients' Hemodynamic parameters.

\begin{tabular}{|c|c|c|c|c|c|c|c|c|c|c|c|c|c|}
\hline \multirow{3}{*}{ Items } & \multicolumn{6}{|c|}{ Control group $(n=30)$} & \multicolumn{6}{|c|}{ Study group $(n=30)$} & \multirow{3}{*}{$\begin{array}{c}\text { P- } \\
\text { value }\end{array}$} \\
\hline & HR & CVP & $\begin{array}{c}\text { SPO } \\
2\end{array}$ & $\mathbf{R} \mathbf{R}$ & $\mathrm{Co}_{2}$ & Bp & HR & CVP & $\begin{array}{c}\text { SPO } \\
2\end{array}$ & $\mathbf{R} \mathbf{R}$ & $\mathrm{Co}_{2}$ & Bp & \\
\hline & $\begin{array}{c}\text { Mea } \\
\text { n } \\
\pm \text { SD }\end{array}$ & $\begin{array}{c}\text { Mea } \\
\text { n } \\
\pm \text { SD }\end{array}$ & $\begin{array}{c}\text { Mea } \\
\text { n } \\
\pm \text { SD }\end{array}$ & $\begin{array}{c}\text { Mea } \\
\text { n } \\
\pm \text { SD }\end{array}$ & $\begin{array}{c}\text { Mea } \\
\mathbf{n} \\
\pm \text { SD }\end{array}$ & $\begin{array}{c}\text { Mea } \\
\text { n } \\
\pm \text { SD }\end{array}$ & $\begin{array}{c}\text { Mea } \\
\text { n } \\
\pm \text { SD }\end{array}$ & $\begin{array}{c}\text { Mea } \\
\text { n } \\
\pm \text { SD }\end{array}$ & $\begin{array}{c}\text { Mea } \\
\text { n } \\
\pm \text { SD }\end{array}$ & $\begin{array}{c}\text { Mea } \\
\text { n } \\
\pm \text { SD }\end{array}$ & $\begin{array}{c}\text { Mea } \\
\text { n } \\
\pm \text { SD }\end{array}$ & $\begin{array}{c}\text { Mean } \pm S \\
\text { D }\end{array}$ & \\
\hline $\begin{array}{l}1^{\text {st }} \text { day } \\
\text { before }\end{array}$ & $\begin{array}{l}103.8 \\
\pm 22.7\end{array}$ & $\begin{array}{c}12.4 \pm \\
7.1\end{array}$ & $\begin{array}{c}92.4 \pm \\
15.1\end{array}$ & $\begin{array}{c}27.6 \pm \\
5.95\end{array}$ & $\begin{array}{l}42.7 \\
\pm 3.2\end{array}$ & $\begin{array}{c}122.3 \\
\pm 15.0 \\
76.3 \pm \\
10.6\end{array}$ & $\begin{array}{l}100.6 \\
\pm 18.6\end{array}$ & $\begin{array}{c}11.9 \pm \\
4.5\end{array}$ & $\begin{array}{c}97.4 \pm \\
2.8\end{array}$ & $\begin{array}{c}29.9 \pm \\
9.7\end{array}$ & $\begin{array}{l}41.8 \\
+5.0\end{array}$ & $\begin{array}{c}116.0 \pm 20 \\
.6 \\
74.6 \pm 12 \\
7\end{array}$ & $\begin{array}{c}0.06 \\
2\end{array}$ \\
\hline $\begin{array}{l}\text { During } \\
\text { sedatio } \\
\mathrm{n}\end{array}$ & $\begin{array}{l}110.6 \\
\pm 27.4\end{array}$ & $\begin{array}{c}30.2 \pm \\
47.2\end{array}$ & $\begin{array}{c}92.1 \pm \\
16.2\end{array}$ & $\begin{array}{c}26.0 \pm \\
5.7\end{array}$ & $\begin{array}{l}40.2 \\
\pm 3.8\end{array}$ & $\begin{array}{c}113.0 \\
\pm 16.6 \\
74.6 \pm \\
11.7\end{array}$ & $\begin{array}{c}92.5 \pm \\
29.9\end{array}$ & $\begin{array}{c}10.5 \pm \\
2.7\end{array}$ & $\begin{array}{c}98.6 \pm \\
1.8\end{array}$ & $\begin{array}{c}26.6 \pm \\
5.2\end{array}$ & $\begin{array}{l}38.4 \\
\pm 3.7\end{array}$ & $\begin{array}{c}116.0 \pm 15 \\
.2 \\
71.3 \pm 8.9\end{array}$ & $\begin{array}{c}0.09 \\
0\end{array}$ \\
\hline $\begin{array}{l}\text { After } \\
\text { withdr } \\
\text { awal }\end{array}$ & $\begin{array}{l}104.0 \\
\pm 24.7\end{array}$ & $\begin{array}{c}12.9 \pm \\
6.8\end{array}$ & $\begin{array}{c}90.0 \pm \\
17.0\end{array}$ & $\begin{array}{c}27.3 \pm \\
5.7\end{array}$ & $\begin{array}{l}40.2 \\
\pm 3.8\end{array}$ & $\begin{array}{c}116.8 \\
\pm 17.4 \\
75.6 \pm \\
11.9\end{array}$ & $\begin{array}{l}105.2 \\
\pm 16.7\end{array}$ & $\begin{array}{c}11.9 \pm \\
5.0\end{array}$ & $\begin{array}{c}98.3 \pm \\
1.6\end{array}$ & $\begin{array}{c}28.7 \pm \\
5.2\end{array}$ & $\begin{array}{l}39.6 \\
\pm 4.1\end{array}$ & \begin{tabular}{|c}
$115.3 \pm 17$ \\
.7 \\
$73.0 \pm 12$. \\
8
\end{tabular} & $\begin{array}{c}0.04 \\
7 *\end{array}$ \\
\hline
\end{tabular}

One way ANOV

* Statistical significant differences $(p<0.05)$

Table (6) pressent distribution of patients out comes and complication.

\begin{tabular}{|l|c|c|c|c|c|}
\hline \multicolumn{1}{|c|}{ Complications and out comes } & $\begin{array}{c}\text { Control } \\
\mathbf{N = 3 0}\end{array}$ & $\mathbf{\%}$ & $\begin{array}{c}\text { Study } \\
\text { N=30 }\end{array}$ & \% & P-value \\
\hline Re sedation \&over sedation & 8 & 26.6 & 5 & 16.6 & $0.041^{*}$ \\
\hline Trach ostomy & 2 & 6.6 & 1 & 3.3 & $0.023^{*}$ \\
\hline Mortality & 10 & 33.3 & 5 & 16.6 & $0.034^{*}$ \\
\hline $\begin{array}{l}\text { Day Icu staying } \\
\text { Mean } \pm \text { SD }\end{array}$ & \multicolumn{3}{|c|}{$\mathbf{4 6 . 0 \pm 2 2 . 1}$} & \multicolumn{3}{|c|}{} & \\
\hline
\end{tabular}

One way ANOVA

* Statistical significant differences $(p<0.05)$

Table (1): Illustrates socio demographic and clinical data of study and control group: Regarding age $65.7 \%$ of control group aged from 41 to 60 years and $60.0 \%$ of study group aged from 21 to 40 years. Regarding sex. Most of control and study group were male. Regarding chronic diseases more than half of control and study group had no chronic diseases. Regarding surgical performance Two third of them hadn't any surgical performance. Regarding the degree of sedation56.7\% of control group had moderate degree of sedation and $66.7 \%$ of study group had moderate degree of sedation. Regarding the aims of sedation. $46.7 \%$ of control group had Mechanical ventilation aim of sedation but at study group $40.0 \%$ of them had pain as aim of sedation.

Figure (1): Percentages distribution of studied patients according to age: This figer show statistical signifficance difference between groups according to age.

Figure (2): Percentages distribution of studied patients according to surgical performance: This figer show statistical signifficance difference between groups according to surgical performance.
Table (2): Shows behavioral pain scale assessment before starting and during the second day and evaluation after weaning or stopping sedation in the third day there was a statistical difference between groups regarding BPS scale with $\mathrm{p}$-value (0 .001 and 0.049 and 0.028 respectively).

Table (3): Shows Richmond agitation sedation scale assessment before starting sedation and during the second day and evaluation after the third day from withdrawal of sedation with $\mathrm{p}$-value $(0.359,0.001$ and 0.005 respectively).

Table (4): Shows ICU screening checklist assessment for delirium monitoring , There was statistical difference between groups regarding patients' ICU screening check list for delirium at starting sedation and during the second day and after the third with p-value ( 0.087 and 0.001 and 0.006 respectively).

Figure (3): Correlation between patients' behavioral pain scale and Richmond agitation sedation scale related to type of sedation This figer show Statistical significance difference between behavioral pain scale 
and Richmond agitation sedation scale related to type of sedation

Table (5): This table shows that; there was a statistical difference between groups regrading patients' hemodynamic parameters at $3^{\text {rd }}$ time with $\mathrm{p}$ value $(0.062,0.090$ and 0.047 respectively).

Table (6): Show there was a statistical difference between the study group and control group in patients out come and complication after sedation.

\section{Discussion}

Sedation has been widely and liberally used in critically ill patients, since the earliest days of intensive care units (ICUs), largely to facilitate uncomfortable mechanical ventilation. (Murray \& Bloomfield, (2008) \& Reade \& Finfer, 2014)

Critical patients are often anxious, agitated, confused, in pain, caused by immobility, injury and/or wounds, and therefore by the adverse environment that surrounds the ICUs. Often there is need to initiate analgesia and sedation as a way to reduce discomfort. In this context, therapeutic, pharmacological and environmental behavior should be considered in order to minimize responses to the environment and to stimuli. (Dantas, et al., 2016).

Nurses are responsible for monitoring the depth of sedation, titrating the infused drugs and monitoring their effects, while maintaining the sedation at a pretargeted level. Protocols or guidelines may assist ICU nurses in making effective clinical decisions, but the effectiveness of sedation administration is still largely dependent on nurse-related factors such as knowledge, skill, experience and confidence. These factors contribute to inappropriate implementation of protocols and affect adherence to evidence-based sedation practices (Ramoo, et al.., 2014)

The present study aim to evaluate the impact implementing of nursing guideline on critically patients outcomes regarding sedations in ICU at Assiut University Hospitals.

One of the main reasons for treating patient in intensive care unit is that they need ventilator support usually by sedation and endotracheal intubation continuous infusion of sedatives and analgesia prolongs ventilators time, increase in which can in it self be harmful. Optimal management of sedation can there for both improve the quality of care and reduce the duration of need for intensive care. (Murray $\&$ Bloomfield, 2008) \& (Brattebøand Hofoss, 2017). This discussion will cover the main result findings as follow:

\section{Socio-demographic characteristics of studied group}

Based on the results of the present study; more than half of study group aged from 21 to 40 years but control group aged from 41 to 60 years, Most of control and study group were male . This agrees with (Azizaet al.., (2011) \& Tai, et al., 2017). Patient related factors like age, gender and weight are important parameters that determine the effective dose of sedatives and analgesics used during anesthesia. As such the target controlled infusion system for propofol, routinely used in the critically care units takes into account both age and gender

Regarding the aim and type of sedation

Main aims of sedation for patients for both in the present study were for pain,mechanical ventilation, and agitation , most common type was (midazolam) which usually adjusts by the physican to give moderate level of sedation majority of studied patients had moderate type of sedation. One third of control group had mechanical ventilation aim of sedation but at study group had pain. The most type of sedatives used in the study was midazolam this which available in the present setting of study, this agree with (Patel, et al., 2014). Who said that midazolam is a popular drug used for sedating mechanically ventilated patients in the intensive care unit (ICU).

(Mitchell et al., 2011) mentioned that Two third of them hadn't any surgical performance who take the sedation for pain only.

In- spit (Burns et al., 1992) mentioned that there is no single desirable depth of sedation or sedative regimen appropriate for all patients. Titration of the sedative dosages and adjustment of the sedative regimen should be done continuously, as dictated by repeated assessments of the patient's needs and current level of sedation.

This result disagree with (Khan, Z., 2017) who mentions that mild sedation is more favorable because it didn't cause sever complication.

Wøien et al., (2014) mentioned that; identification of the critically ill patient's need for pain relief and correct level of sedation decreases the risk of complications and reduces the length of stay it was matching with our result about the main cause of sedation administration is pain reliever.

Walsh et al., (2016). Said that the main cause of sedation uses is the average change in mechanically ventilation by impedance following sedation was less half of studied patients.

(Marquis et al., 2017) mentioned that one third of respondents stated that they routinely used sedative agents in mechanically ventilated patients majority of the respondents stated that they used sedative agents to "suppress excessive or dangerous motor activity" and more than half said they used them "to promote sleep". Since these studies, there has been a paradigm change in our approach to sedation of ICU patients. Moderate sedation is delivered in a number of settings. The reasons for sedation may vary, but the 
Joint Commission (2011) requires that the patient receive the same level of care regardless of the practice setting.

Additionally, moderate sedation has been used as an adjunct to local anesthesia. A vast number of procedures that are uncomfortable and/or painful can be performed safely using moderate sedation. That agree with the present study" the majority of studied patients had moderate type of sedation".

Multiple studies have reported the harmful short and longer-term effects of over sedation. Moreover, advances in technology have enabled development of ventilators that synchronize much better with a patient's own respiratory efforts reducing the need for deep sedation in patients receiving mechanical ventilation. Barr, et al., (2013) the latest guidelines from the American College of Critical Care Medicine recommend that "sedative medications be titrated to maintain a light rather than a deep level of sedation in adult ICU patients, unless clinically contraindicated".

\section{Regarding the behavioral pain scale}

The present study mentioned that the pain score decreased in study group after implementing nursing guide lines application rather than control group thiswith the same line with (Barr et al., 2013) who mentioned that the guidelines provide a road map for developing integrated, evidence-based, and patientcentered protocols for preventing and treating pain, agitation, and delirium in critically ill patients.

The study revealed that there is a statistical significance difference between study and control groups regarding behavioral pain scale before and after sedation study group treatment also there is a statistical difference between groups regarding behavioral pain scale after the third day from sedation treatment discontinuation this disagree with (Rijkenberg, et al., 2015) \& (Syan et al., 2017) who mentioned that the behavioral pain scale remained unchanged after using sedation for pain relive patients.

The present study demonstrated that there is a statistical significance difference between groups before and during sedation treatment also after sedation treatment cessation regarding behavioral pain scale this agree with (Vasilevskis, et al., 2016) who suggest that pain management guidelines had positive effect on behavioral pain scale.

Regarding Richmond agitation sedation scale (RASS)

The present study demonstrated that there was a statistical difference between groups regarding Richmond agitation sedation scale. This agreed with (Mehany et al., 2016) \& (Vasilevskis, et al., 2016). Who suggested that Delirium resolved faster with the duration of mechanical ventilation and ICU length of stay pain scale Also the RASS analyzed had moderate sensitivity and very high specificity for the detection of delirium superimposed on dementia (DSD).

The study also found that there was a statistical significance difference between groups regarding Richmond agitation sedation scale after withdrawal of sedation.

Anderson, et al., (2016) dis agree with this research results in his study " Concordance Between Bedside Nursing And Protocolized Sedation Assessments In Medical Intensive Care Unit Patients " which showed that Richmond agitation sedation scale was not significantly associated with patient's status and ICU out come.

Regarding patients' ICU screening check list for delirium

There was a statistical difference between groups regarding patients' ICU screening check list for delirium during sedation administration may attributed to positive effect regarding nursing guideline on study group.

Accumulating evidence suggests that the management of sedation and delirium can have an important effect on the outcomes of patients who are treated in ICUs. Currently available data suggest that the best outcomes are achieved with the use of a protocol in which the depth of sedation and the presence of pain and delirium are routinely monitored, pain is treated promptly and effectively, the administration of sedatives is kept to the minimum necessary for the comfort and safety of the patient, and early mobilization is achieved whenever possible (Reade \& Finfer, 2014).

Murray \& Bloomfield, (2008) \& Klouwenberg, et al., (2014) founded that When delirium was assessed 48 hours after sedation was discontinued, there were no significant differences among the groups and mention that " Regardless of the cause and the underlying pathophysiology, delirium is now recognized as a frequent and serious event in critically ill patients specially who receiving sedation". That finding is against the study result which found a significant difference between the study and control groups regarding the severity of delirium.

With reference to ICU intensive screening check list assessment for dilirum, the study shows that, there was a statistical difference between groups regarding patients' ICU screening check list for delirium and after sedation. Regardless of the cause and the underlying pathophysiology, delirium is now recognized as a frequent and serious event in critically ill patients specially who receiving sedation Klouwenberg, et al., (2014) found that When delirium was assessed 48 hours after sedation was discontinued, there were no significant differences 
among the groups.that is against the study result which found asignifecant defference between the study and control groups regarding the sevserity of delirium.

Hughes et al., (2013) demonstrated that; agitation and anxiety occur frequently in critically ill patients and are associated with adverse clinical outcomes, to which sedatives are commonly administered.

This was in line with Scholtens, et al., (2017) in his study titled "Variability of delirium motor subtype scale-defined delirium motor subtypes in elderly adults with hip fracture" who reported that, the hyperactive and hypoactive motor subtypes were associated with change. This could indicate a treatment effect, because individuals with hyperactive delirium are often prescribed psychopharmaca and thus might change to the hypoactive motor subtype because of sedation. The fact that a trend for general anesthetics was observed in the current study is in line with this study.

Growing evidence reveals the majority of critically ill patients are at risk for developing two common, dangerous, and potentially iatrogenic conditionswhich are intensive care unit (ICU) delirium and weakness. ICU-acquired delirium and weakness not only influence a patient's ability to survive critical illness, but are also associated with poor long- term physical, functional, and cognitive outcomes (Balas et al., 2014).

Correlation between patients' behavioral pain scale and Richmond agitation sedation scale related to aim of sedation. Our study showed that aim of sedation was an effective factor of the level of pain. This in the same line with Payen, et al., (2001) in his study titeled "Assessing pain in critically ill sedated patients by using a behavioral pain scale "who inform that the type of sedation effect on behavioral pain scale.

In present study a statistical difference between groups regarding patients' hemodynamic parameters at $3^{\text {rd }}$ day of sedation administration. It showed that the sedatives affected on the patient's parameters and hemodynamic status it was in comparative with MacLaren, et al., (2007).

We used oxygen saturation of hemoglobin ( $\mathrm{SpO} 2)$ in present study, which is an indicator for oxygenation. While sedation types did not show any differences in $\mathrm{SpO} 2$ values before administration and 1stday, the value of $\mathrm{SpO} 2$ in study groups were higher than the values in the control group after one day. While all sedative agents increased SPO2 levels after third day of sedation, Sedative agents increased the SPO2 level as expected.

Regarding the frequency of complication and outcomes
The frequency complication for patients who was exposed to the designed nursing guide line for sedative critically ill patient protocol was lesser than among control group of patients and the number of improved patients were higher in study group rather than control.

In the same line with (Shafer, 1998) who mentioned that Continuous infusion of midazolam provides an effective sedation in the ICU with few complications overall, especially when the dose is titrated.

But Sedative drugs in the critically ill may contribute to increased morbidity, and possibly mortality according to (Watt, 1984) \& (Bowdle, 2017).

Multiple studies have examined the depth of sedation in relation to several clinical outcomes: duration of mechanical ventilation, ICU length of stay, physiologic/psychological stress. Results of these studies suggest that maintaining critically ill patients at a light level of sedation is favorable compared to deep sedation, yet not all studies agreed. (Varndell, et al., 2015).

\section{Conclusion \& Recommendation}

Based on the result of this study, it could be concluded that: Implementing of nursing guidelines about sedation is significantly effective in preventing complication of sedatives in critically patients (tracheostomy, mortality, ICU staying, and over sedation) and improve patients' outcomes.

\section{Based on finding of this study, it recommended} that:

- Emphasize to utilize the nursing guidline about sedation in the critically care units

- Equip intensive care units with simple illustrated book let about sedation nursing guidelines.

- Reapply this research on a larger probability sample acquired from different geographical areas in Egypt for generalization.

\section{References}

1. Anderson, C., Jolley, S., Johnson, J., \& DeBoisblanc, B., (2016): Concordance between Bedside Nursing and Protocolized Sedation Assessments in Medical Intensive Care Unit Patients. In C50. CRITICAL CARE: DELIRIUM AND SEDATION IN THE ICU (pp. A5258A5258). American Thoracic Society.

2. Aziza E., Nahad E., Nabila A., \& Wael, S., (2011): Comparative Study of Ozonated Olive Oil Ointment versus Conventional Dressing Methods on the Healing of Grade I Diabetic Foot Ulcers. Researcher, 3(8):16-30]. (ISSN: 1553-9865). http://www.sciencepub.net.

3. Balas, M., Vasilevskis, E., Olsen, K., Schmid, K., Shostrom, V., Cohen, M., \& Stothert, J., (2014): Effectiveness and safety of the awakening 
and breathing coordination, delirium monitoring/management, and early exercise/mobility (ABCDE) bundle. Critical care medicine, 42(5), 1024.

4. Barr J., Fraser G., Punlillo, K., EIy, E., Gelinas, C., Dasta J., \& Coursin, D., (2013): Clinical Practice Guidelines for the Management of Pain, Agitation, and Delirium in Adult Patients in the Intensive Care Unit. Crit Care Med.; 41(1):278.

5. Barr J., Fraser G., Punlillo, K., EIy, E., Gelinas, C., Dasta J., \& Coursin, D., (2013): Clinical practice guidelines for the management of pain, agitation, and delirium in adult patients in the intensive care unit. Critical care medicine, 41(1), 263-306.

6. Brattebo G., Hofoss D., Flaatten H., Muri A., Gjerde S., Plsek P., (2017): Effect of a scoring system and protocol for sedation on duration of patients' need for ventilator support in a surgical intensive care unit; 324(7350):1386-9.

7. Bowdle, T., (2017): Is Etomidate Sedation Associated With Excess Mortality in Intensive Care Unit Patients? What Is the Evidence? Anesthesia \& Analgesia, 125(2), 713

8. Burns A., Shelly M., Park G., (1992): The use of sedative agents in critically ill patients. Drugs; 43:507-515

9. Dantas, K., Costa, I., Fernanda, M., (2016): Alves Ferreira Gonçalves1Characterization of the sedation and analgesia in Intensive Care Unit: an observational vol15, NO2.2016 1Online Brazilian Journal

studyhttp://www.objnursing.uff.br/index.php/nurs ing/issue/view/66.

10. Girard T., Kress J., Fuchs B., (2013): Efficacy and safety of a paired sedation and ventilator weaning protocol for mechanically ventilated patients in intensive care (awakening and breathing controlled trial): a randomized controlled trial. Lancet. 371: 126-34.

11. Hariharan U., \& Garg, R., (2017): Sedation and Analgesia in Critical Care Volume 7 Issue 3 2017, Journal of Anesthesia \& Critical Care: Open Access Received: January 06, 2017 | Published: March 02

12. Hogarth K., \& Hall J., (2004): management of sedation in mechanically ventilated patient's Lippincott Williams Q wilkines 1070-5295. Correspondence to Jesse Hall, MD, University of Chicago Hospitals, 5841 South Maryland Ave, MC 6026, Chicago, IL 60637, USA

13. Hughes, C., Girard, T., \& Pandharipande, P., (2013). Daily sedation interruption versus targeted light sedation strategies in ICU patients. Critical care medicine, 41(9), S39-S45.
14. Khan, Z., (2017): Sedation and monitoring. Chapman \& Nakielny's Guide to Radiological Procedures E-Book, 385

15. Klouwenberg, P., Zaal, I., Spitoni, C., Ong, D., Van Der Kooi, A., Bonten, M., \& Cremer, O., (2014): The attributable mortality of delirium in critically ill patients: prospective cohort study. bmj, 349, g6652

16. MacLaren, R., Forrest, L., \& Kiser, T., (2007): Adjunctive dexmedetomidine therapy in the intensive care unit: a retrospective assessment of impact on sedative and analgesic requirements, levels of sedation and analgesia, and ventilatory and hemodynamic parameters. Pharmacotherapy: The Journal of Human Pharmacology and Drug Therapy, 27(3), 351-359.)

17. Grap, M., Cindyl, M., Humilton A., \& Curtis N., Sessler (2012): Sedation in Adults receiving mechanical ventilation physiological and comfort guideline, American Journal of critical care may volum 21 No 3.

18. Marquis, J., Rim, F., Marquis, M., Hotel, J., \& York, F., (2017): aba future midyear and annual meetings year midyear annual. lea, 2016, 6.

19. MacLaren, R., Forrest, L., \& Kiser, T., (2007): Adjunctive dexmedetomidine therapy in the intensive care unit: a retrospective assessment of impact on sedative and analgesic requirements, levels of sedation and analgesia, and ventilatory and hemodynamic parameters. Pharmacotherapy: The Journal of Human Pharmacology and Drug Therapy, 27(3), 351-359.

20. Mehany, M., Elaal, E., \& Kamel, M., (2016): Effect of Specific Nursing Intervention on Respiratory Status of Chronic Obstructive Pulmonary Disease (COPD) During Acute Exacerbation, IOSR Journal of Nursing and Health Science (IOSR-JNHS) e-ISSN: 23201959.p- ISSN: 2320-1940 Volume 5, Issue 2 Ver. I, PP 77-84 www.iosrjournals.org. DOI: 10.9790/1959-05217784

21. Mitchell P., Fink, Jean-Louis Vincent, Edward Abraham, Patrick Kochanek, Frederick A., Moore, (2011): Textbook of Critical Care EBook, 6th ed, Elsevier Health Sciences companyP.P. 1366

22. Morgan, R., (2007): over view of sedation in intensive care, nursing training program.journal of anisthology news. Nursing training program: Sedation in the intensive care setting. McMahon Publishing.NY, NY.

23. Murray M., Abd Bloomfield E., (2008): sedation and neuromuscular blockade, Pharmacology, Nutrition, Toxicology, and the Environment, chapter 63, Section VII, P.p. 17:57, 962. 
24. Norman, C., (2014): $\quad$ Moderate Sedation/Analgesia Release Date: 08/01/2014. (valid through December 12, 2017); California, BRN Provider \#CEP9784; California, LVN Provider \#V10662; California, PT Provider \#V10842; Florida, Provider \#50-2405; Iowa, Provider \#295; Kentucky, Provider \#7-0054 through 12/31/2017.

25. Patel, S., Poston, J., Pohlman, A., Hall, J., \& Kress, J., (2014): Rapidly reversible, sedationrelated delirium versus persistent delirium in the intensive care unit. American journal of respiratory and critical care medicine, 189 (6), 658-665

26. Pandharipande, P., Patel, M., Barr, J., (2014): Management of pain, agitation, and deliriumin critically ill patients114 polskie archiwum medycyny wewnętrznej,; 124

27. Richard R., (2013). Evidence - based ICU sedation guideline main medical center port land main USA.Tufts University School of Medicine ... Portland, Maine USA. 1 ... GRADE-based Guidelines ACCM.... Propofol and the development of delirium in adult ICU patients (C).27. https://criticalcarecanada.com.

28. Reade, M., \& Finfer, S., (2014): Sedation and delirium in theintensive care unit. New England Journal of Medicine, 370(5), 444-454

29. Rijkenberg, S., Stilma, W., Endeman, H., Bosman, R., \& Oudemans-van Straaten, H., (2015): Pain measurement in mechanically ventilated critically ill patients: behavioral pain scale versus critical-care pain observation tool. Journal of critical care, 30(1), 167-172

30. Scholtens, R., van Munster, B., Adamis, D., Jonghe, A., Meagher, D., \& de Rooij, S., (2017): Variability of delirium motor subtype scale-defined delirium motor subtypes in elderly adults with hip fracture: A longitudinal study. Journal of the American Geriatrics Society, 65(2)

31. Shafer, A., (1998): Complications of sedation with midazolam in the intensive care unit and a comparison with other sedative regimens. Critical care medicine, 26(5), 947-956

32. Sherman, R., de Beer T., (2014): Adult critical care guidelines for sedation and analgesia in the intubated patient by Nottingham university hospital. Consultation process Critical Care Cross Town Protocol and Guideline Group, Critical Care Directorate. Adult Critical Care Department, Nottingham University Hospitals NHS Trust.

33. Stites, M., (2013): observational pain scales in critically ill adults .critical care nurse vol 33 No.3,American Association of Critical Care Nurses doi:http \\dx.doi .org\10.4037\ccn2013804
34. Syan S., Abed El-Kreem H., Ibrahim I., \& Mohammed E., (2017): Impact Of Developed Standards Of Care On Post-Operative Outcomes In Children With Inguinal Hernia At Assiut Children University Hospital, The Malaysian jurnal of Nursing, Volume-9, Issue 1, 2017, Pages $3-15$.

35. Tai, B., Remani, D., Phua, J., (2017): Age related inverse dose relation of sedatives and analgesics in the intensive care unit Published: September 28, https://doi.org/10.1371/ journal. pone. 0185212 .

36. The Joint Commission, (2011): Comprehensive Accreditation Manual for Hospitals: The Official Handbook. Oakbrook Terrace, IL: Joint Commission Resources.

37. Varndell, W., Elliott, D., \& Fry, M., (2015): Assessing, monitoring and managing continuous intravenous sedation for critically ill adult patients and implications for emergency nursing practice: A systematic literature review. Australasian Emergency Nursing Journal, 18(2), 59-67.

38. Vasilevskis, E., Pandharipande, P., Graves, A., Shintani, A., Tsuruta, R., Ely, E., \& Girard, T., (2016): Validity of a modified sequential organ failure assessment score using the Richmond agitation-sedation scale. Critical care medicine, 44(1), 138

39. Ramoo, V., Abdullah, K., Tan, P., Wong, L., \& Piaw Y., (2014): ChuaIntervention to improve intensivecare nurses' knowledge of sedationassessment and managementdoi: 10.1111/ nicc.12105@ British Association of Critical Care Nurses. Vol 21 No 5 287@ British Association of Critical Care Nurses. Vol 21 No 5287

40. Rowe, K., Fletcher, S., (2017): Sedation in the intensive care unit Copyright (C) 2017 Oxford University Press, the British Journal of Anaesthesia Ltd, Anaesth Crit CarePain (2008)8(2):50-55. Available at https://doi.org/ 10.1093/bjaceaccp/mkn005.

41. Walsh, T., Kydonaki, K., Lee, R., Everingham, K., Antonelli, J., Harkness, R., \& Davidson, A., (2016): Development of process control methodology for tracking the quality and safety of pain, agitation, and sedation management in critical care units. Critical care medicine, 44(3), 564-574.

42. Watt I., Ledingham I., (1984): Mortality amongst multiple trauma patients admitted to an intensive therapy unit. Anaesthesia; 39:973-981

43. Wøien, H., Værøy, H., Aamodt, G., \& Bjørk, I., (2014): Improving the systematic approach to pain and sedation management in the ICU by using assessment tools. Journal of clinical nursing, 23(11-12), 1552-1561. 